\title{
Active Contour Models for Shape Description Using Multiscale Differential Invariants
}

\author{
Julia A. Schnabel and Simon R. Arridge \\ Department of Computer Science \\ University College London
}

\begin{abstract}
Classic curvature-minimizing active contour models are often incapable of extracting complex shapes with points of high curvature. This paper presents a new active contour model which overcomes this problem and which can be applied to image segmentation as well as shape description in order to allow for quantitative and qualitative studies of shape measurements at multiple scales. Multiscale differential operators, which are invariant to linear intensity transformations such as contrast or brightness adjustments and independent of coordinate transformations, are integrated into the model's spline energy functional. Whereas the image intensity gradient attracts the spline contour to image features, the isophote curvature of the image intensity function is used for matching the contour curvature. This novel curvature matching approach appears to be very useful for the extraction of very complex and strongly curved objects such as brain contours, results of which will be presented in this paper.
\end{abstract}

Keywords: Active contour models, shape description, multiscale representation, curvature

\section{Introduction}

Active contours or snakes were first introduced by Kass et al. [7, 16]. They are a segmentation tool based on minimizing the energy of a spline contour in terms of internal and external constraints. The internal constraints determine the autonomous shape of the model, while the external constraints draw the model towards image features. The contour described by the active contour model is given by a vector $\mathbf{v}(s)=(x(s), y(s))$ with arc length parameter $s$. The energy functional of the contour is defined as

$$
E_{\text {snake }}^{*}=\int_{0}^{1} E_{\text {snake }}(\mathbf{v}(s)) d s=\int_{0}^{1} E_{\text {intern }}(\mathbf{v}(s))+E_{\text {image }}(\mathbf{v}(s)) d s,
$$


where $E_{\text {intern }}$ is the internal energy of the contour with respect to elastic deformations and bending of the active contour. The internal energy is defined as

$$
E_{\text {intern }}(\mathbf{v}(s))=\alpha(s)\left|\mathbf{v}_{s}(s)\right|^{2}+\beta(s)\left|\mathbf{v}_{s s}(s)\right|^{2},
$$

where the first order or elasticity term, $\mathbf{v}_{\mathbf{s}}(s)$, makes the snake behave like a membrane, and the second order or bending term, $\mathbf{v}_{s s}(s)$, makes the snake behave like a thin plate.

The image energy term $E_{\text {image }}$ pulls the active contour towards features in the image and can be defined as suggested by Kass et al. [7] using the image intensity gradient:

$$
E_{\text {image }}(\mathbf{v}(s))=-|\nabla I(x, y)|^{2}
$$

During the optimization process, the active contour is deformed with respect to the features to be localized. There are several optimization approaches for active contour models, including a variational approach by Kass et al. [7], dynamic programming by Amini [1], the greedy algorithm by Williams and Shah [17], genetic algorithms by Cootes et al. [6], and stochastic relaxation techniques by Rueckert [13]. For the purpose of this paper, only a local deformation of the active contour is desired, hence a refined greedy algorithm providing an efficient local optimization was found suitable.

This paper proposes a new approach using active contour models as multiscale shape descriptors. Using scale space continuation in active contour models provides the ability to capture image features at the adequate scale. Using an initial active contour model, several implicit optimization processes with differently regularized energy functions with respect to scale are performed. The results are formulated in a multiscale hierarchy and are qualitatively and quantitatively evaluated. In the following sections, we will present this refined model.

\section{Curvature Matching Process in Scale Space}

Over the last few years, multiscale approaches in image analysis have proved to be useful in terms of describing images at varying levels of resolution. The fundamental concept of multiscale image processing was developed by Koenderink [8] and Witkin [18]. The underlying image can be represented by a family of images on various levels of inner spatial scale. These are obtained by convolution with the Gaussian kernel as the lowest order, rescaling operator, and its linear partial derivatives. A continuous scale space is constructed to enable local image analysis in a robust way, while at the same time global features are captured through the extra scale degree of freedom. Multiscale differential invariants, as presented by Romeny et al. [14, 15], are true image descriptors that resemble the receptive field profiles in the human front-end visual system and are invariant with respect to the chosen coordinate system and linear transformations of the image intensity.

The curvature term in the internal energy term in equation (2) minimizes the bending of the active contour model assuming that the object of interest has a 

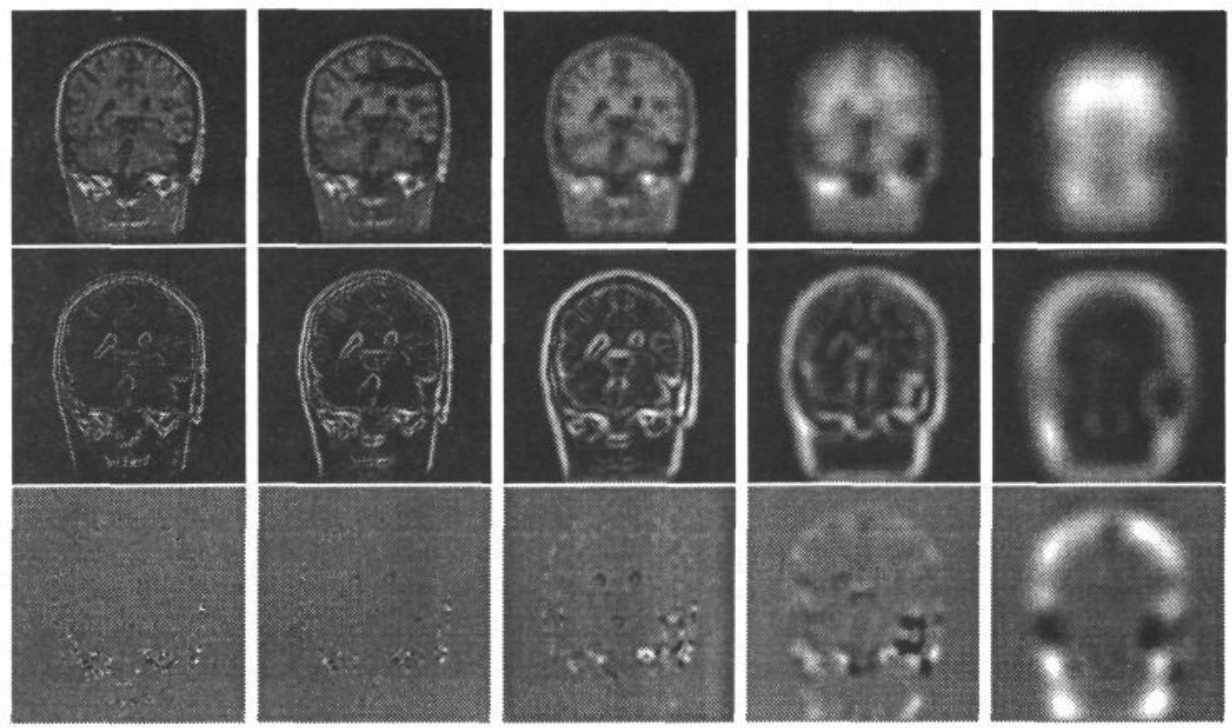

Figure 1: From top to bottom: Gaussian smoothed image, gradient image and isophote curvature image for (from left to right) $\sigma=1, \sigma=2, \sigma=4, \sigma=8$ and $\sigma=16$.

smooth contour with low curvature. However, this does not take into account that some objects of interest may have contour parts of high curvature. Cohen [5] proposed to use implicit expressions of material densities by optimizing bending densities depending on the metric as well as on first and second order variations of the gradient potential along the model. Rougon [12] refined this approach by using oriented anisotropic adaptive material parameters that explicitly depend on the parameterized contour function. Yet none of these approaches takes the underlying image intensity curvature into account. This paper proposes the integration of a matching process between the image and contour curvature into the active contour model, in order to adapt the curvature of the active contour to the curvature of the image intensity function. This is achieved by minimizing the deviation of the isophote image intensity curvature from the contour curvature.

\subsection{Image Curvature}

In order to compute the curvature of an image with respect to scale (or tolerance with which the image is processed), the image is convolved (blurred) with a small neighbourhood-averaging kernel. Blurring the image with increasingly larger kernels produces larger-scale versions of the image. It has been shown [8] that the Gaussian $G$ or derivatives of the Gaussian as solutions to the diffusion equation are the only reasonable scaling operators:

$$
G\left(x, y ; \sigma_{i}\right)=\frac{1}{2 \pi \sigma_{i}^{2}} e^{\frac{-\left(x^{2}+y^{2}\right)}{2 \sigma_{i}^{2}}}
$$


where $\sigma_{i}$ is the scale or width of the scaling operator. In order to build a scale space, the scale is sampled exponentially as $\sigma_{i}=\sigma_{0} f^{i}$, where $\sigma_{0}$ is the initial scale and $f$ is the scale-change factor. We will denote the Gaussian smoothed image as $L\left(x, y ; \sigma_{i}\right)$ or just $L$, and its partial derivatives (convolutions of the image with partial derivatives of the Gaussian kernel) with appropriate subscripts. The twodimensional isophote curvature $C\left(x, y ; \sigma_{i}\right)$ can then be computed as:

$$
C\left(x, y ; \sigma_{i}\right)=\frac{2 L_{x} L_{y} L_{x y}-L_{x}^{2} L_{y y}-L_{y}^{2} L_{x x}}{\left(L_{x}^{2}+L_{y}^{2}\right)^{\frac{3}{2}}}
$$

Lindeberg [9] proposes to multiply the curvature by the gradient magnitude raised to some power, $k$, a natural choice being $k=3$, in order to give a stronger response near edges. Examples of a Gaussian blurred image, its image gradient and its isophote image curvature (multiplied by $|\nabla L|^{3 / 2}$ ) for different resolutions are presented in figure 1.

\subsection{Contour Curvatures}

According to Mokhtarian and Mackworth [10, 11], a contour is represented by its discrete contour control points, which can be parameterized in terms of two functions $x(s)$ and $y(s)$ as $\{x(s), y(s)\}$, with $0 \leq s \leq 1$. For closed curves, $x(s)$ and $y(s)$ are periodic functions. The contour curvature $\kappa$ of a planar curve can be expressed in terms of partial derivatives of $x(s)$ and $y(s)$ by

$$
\kappa=\frac{\dot{x}(s) \ddot{y}(s)-\dot{y}(s) \ddot{x}(s)}{\left(\dot{x}(s)^{2}+\dot{y}(s)^{2}\right)^{3 / 2}} .
$$

To obtain descriptions of the curve at varying levels of detail, the functions $x(s)$ and $y(s)$ can be convolved with a one-dimensional Gaussian kernel for varying spatial widths $\sigma$.

A convenient way to represent a contour is using interpolating $C^{2}$ polynomial spline patches, which are very attractive tools for active contour models, as they yield a continuous smooth description of the contour by approximating the set of contour control points. Their analytic curvature can thus be computed not only at the contour control points, but all along the interpolated contour, which is a desirable extension of the classic active contour model. To allow for local control and analytic computation of the spline elasticity and bending, a cubic $C^{2}$ continuous B-spline as presented in [2] can be used for representing the active contour model. Cubic B-splines approximate series of $n$ control points $P_{0} \cdots P_{n-1}, n \geq 3$, with a closed curve consisting of $n$ polynomial curve segments. Each of the curve segments is defined by four of the control points, where curve segment $\mathbf{Q}_{i}$ is defined by the geometric constraints $P_{i-1}, P_{i}, P_{i+1}, P_{i+2}$ and the blending functions and polynomial coefficients:

$$
\mathbf{Q}_{i}(t)=\left[\begin{array}{llll}
t^{3} & t^{2} & t & 1
\end{array}\right] \cdot \frac{1}{6}\left(\begin{array}{rrrr}
-1 & 3 & -3 & 1 \\
3 & -6 & 3 & 0 \\
-3 & 0 & 3 & 0 \\
1 & 4 & 1 & 0
\end{array}\right) \cdot\left[\begin{array}{l}
P_{i-1} \\
P_{i} \\
P_{i+1} \\
P_{i+2}
\end{array}\right]=\left(\begin{array}{l}
x_{i}(t) \\
y_{i}(t)
\end{array}\right)
$$



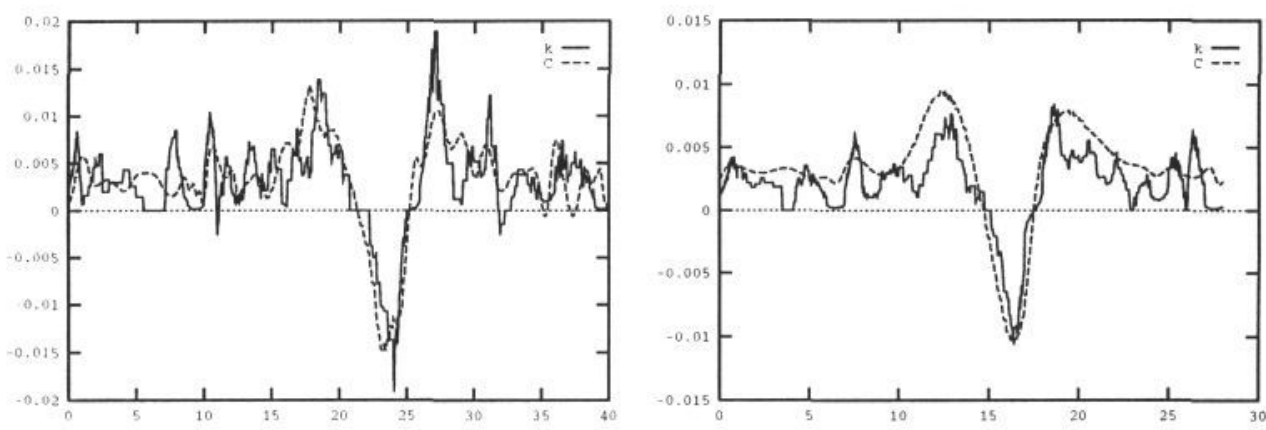

Figure 2: Spline curvature $\kappa$ and isophote image curvature $C$ along the spline for resampling distance $\delta=4, \sigma=4$ (left) and $\delta=8, \sigma=8$ (right).

Differentiating the polynomials defined above allows for analytic computation of the spline curvature as in equation (6) and the spline continuity or elasticity. The linearity of $\ddot{\mathbf{Q}}_{i}(t)$ causes a lack of smoothness for the spline curvature, which could be overcome by using a higher-order spline polynomial. However, as higher-order polynomials can cause unwanted oscillations and zero-crossings, they are not useful for the purpose of this paper. We have found that B-splines prove to be sufficient interpolants in terms of spline curvature and elasticity estimation.

\subsection{Matching process}

In order to match the B-spline curvature of a contour to the underlying isophote image curvature, the B-spline curvature needs to be normalized by the distance of the contour control points. Tests on an artificial image containing an object with one major concavity showed that best matching results can be achieved when the distance $\delta$ between the contour control points corresponds to the spatial width $\sigma$ of the Gaussian kernel for the isophote curvature computation. Increasing the distance between the contour control points achieves a smoothing effect of the spline, as details are suppressed. Thus the B-spline has to be resampled with respect to the scale by inserting new equidistant control points of distance $\delta \approx \sigma$ along the original spline. Figure 2 shows the curvature profiles of the isophote image curvature along the resampled spline as well as the spline curvature for two different $\delta$ and $\sigma$. As can be clearly seen, the range as well as the shape of the image and spline curvatures with respect to zero-crossings, points of inflection and extrema can be easily matched. Thus an integration of the deviation of the spline curvature from the image curvature seems highly appropriate.

B-splines have the following attractive properties which make them very useful for shape representation and analysis as pointed out by Cohen and Wang [4], and thus their application for active contour models is highly appropriate: 
- They are smooth and continuous interpolants of the active contour control points representing the contour curve.

- They are locally controlled, which implies that local changes in shape are reflected by local changes of the B-spline parameters which makes them highly applicable for local, quasi-parallel optimization techniques of the active contour's energy functional such as the greedy algorithm or ICM.

- They have a generative nature, thus the curve can be generated at any detail desired (i.e. by using different sampling rates) with respect to the required image resolution.

- Their explicit polynomial representation allows for analytic differentiation and curvature analysis of the contour which makes the problematic use of discrete approximations of the contour elasticity and bending as investigated by Williams and Shah [17] obsolete.

The curvature matching is integrated into the energy functional of the refined active contour model which is presented in the following section. To allow for a proper matching, the initial active contour is resampled with respect to the scale used for the computation of the isophote image intensity curvature, which leads at the same time to fewer contour control points for higher scales, as it is sufficient to describe the contour with less points at lower image resolutions.

\section{Refined Active Contour Model}

In order to achieve the curvature matching discussed above, the classic energy functional has to be modified with respect to the integration of the image curvature and the whole spline contour. After the presentation of the refined active contour model and its energy terms, the optimization strategy will be explained.

The incorporation of the spline model allows to analytically compute the spline elasticity as well as the spline curvature not only for the contour control points, but all along the spline. The model's internal constraints depend on the resampling distance of the spline control points and thus on the directly related image scale $\sigma$. Thus, the discrete normalized sum of points along the $i$-th spline patch can be computed for the elasticity and bending energy terms by

$$
E_{\text {elas }}\left(\mathbf{Q}_{i} ; \sigma\right)=\frac{1}{N_{i}} \sum_{t=0, \Delta t_{i}}^{t=1} \sqrt{\dot{x}_{i}(t)^{2}+\dot{y}_{i}(t)^{2}}
$$

and

$$
E_{b e n d}\left(\mathbf{Q}_{i} ; \sigma\right)=\frac{1}{N_{i}} \sum_{t=0, \Delta t_{i}}^{t=1}\left|\kappa(t)-C\left(x_{i}(t), y_{i}(t) ; \sigma\right)\right|,
$$

where $N_{i}$ is the length of the spline patch $\mathbf{Q}_{i}$ and the step size for the summation is chosen as $\Delta t_{i}=1 / N_{i}$, and $C$ is the isophote curvature of the image intensity 
function as defined in equation (5). As the sign of the isophote image curvature depends on whether the object is dark on a light background or vice versa, this fact must be included as a priori knowledge. Thus not only the amount of bending of both spline and image curvature can be matched, but the bending behaviour itself is taken into account.

The image energy term is defined analogously by incorporating not only the image gradient, but the Chamfer distance transform image [3] of the zero crossings, $D_{z c}(x, y ; \sigma)$ which creates an attraction potential which draws the model towards edges. The image term is computed like the internal energy terms along the spline contour:

$$
E_{\text {imag }}\left(\mathbf{Q}_{i} ; \sigma\right)=\frac{1}{N_{i}} \sum_{t=0, \Delta t_{i}}^{t=1}\left|\nabla L\left(x_{i}(t), y_{i}(t) ; \sigma\right)\right|^{2}+D_{z c}\left(x_{i}(t), y_{i}(t) ; \sigma\right)
$$

All energy terms are normalized within their local neighbourhood. The complete, weighted energy functional of the $i$-th spline patch of the refined active contour model is a linear combination of the internal and external energy terms:

$$
E_{\text {snake }}\left(\mathbf{Q}_{i} ; \sigma\right)=\alpha E_{\text {elas }}\left(\mathbf{Q}_{i}(s) ; \sigma\right)+\beta E_{\text {bend }}\left(\mathbf{Q}_{i}(s) ; \sigma\right)+\gamma E_{\text {imag }}\left(\mathbf{Q}_{i}(s) ; \sigma\right)
$$

This energy term is not only dependent on $\sigma$ because of the incorporation of the scale space invariants in terms of image curvature, gradient and zero crossings, but also because of the sampling distance $\delta$ of the spline, which is directly related to the spatial width $\sigma$. As the $i$-th spline control point influences both $\mathbf{Q}_{i}$ and $\mathbf{Q}_{i+1}$, the sum of the bending, elasticity and image energy terms of both spline patches has to be computed, thus

$$
E_{\text {snake }}\left(\mathbf{v}_{i}(s) ; \sigma\right)=E_{\text {snake }}\left(\mathbf{Q}_{i}(s) ; \sigma\right)+E_{\text {snake }}\left(\mathbf{Q}_{i+1}(s) ; \sigma\right)
$$

To optimize the presented active contour model with respect to the energy functional defined above, a refined greedy algorithm was developed. While the original algorithm by Williams and Shah [17] performs a sequential optimization and updating of the model, this implementation uses a different, quasi-parallel optimization approach. Within one iteration of the algorithm, every control point $\mathbf{v}_{i}(s)$ is compared to its direct neighbouring pixels in terms of its energy, but only after the iteration the control points are updated to avoid sequential dependence.

\section{Shape Description in Scale Space}

The active contour model with the integrated curvature matching process presented in this paper was used as as method for shape description in order to quantify an object's change of shape by regularizing the energy function according to scale. A magnetic resonance image of the brain, whose invariants in scale have been presented in figure 1, was presegmented to allow for an accurate initialization of the active contour model. A series of implicit segmentation processes and optimizations with differently regularized energy functions with respect to scale and 
spline sampling distance were performed. The obtained optimization results were quantified with classic shape descriptors such as area, perimeter and compactness, and they were compared with the original contour with respect to segmentation errors as well as using the Chamfer distance transform to quantify differences of shape.

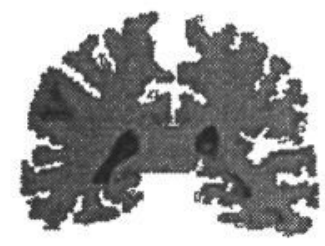

Original image

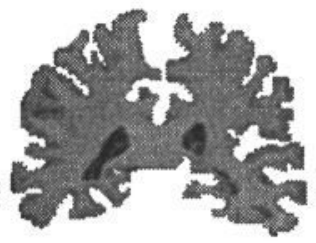

$\sigma=1$

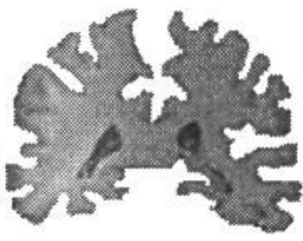

$\sigma=2$

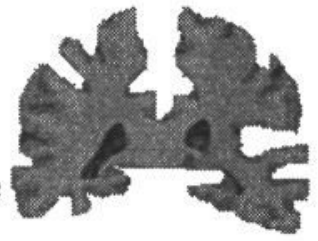

$\sigma=4$

Figure 3: Optimization results for different spatial widths $\sigma$.

\begin{tabular}{|l|c|c|c|r|c|l|l|}
\hline$\sigma$ & Area & Perimeter & Compactness & $E_{\text {abs }}$ & $E_{\text {mean }}$ & $L_{1}$ & $L_{2}$ \\
\hline \hline 1 & 9216 & 4530 & 2226 & 854 & 0.918 & 0.4602 & 0.0110 \\
\hline 2 & 8965 & 4037 & 1817 & 1101 & 0.1183 & 0.5626 & 0.0128 \\
\hline 4 & 8516 & 3373 & 1335 & 1842 & 0.1985 & 1.0176 & 0.0224 \\
\hline 8 & 9639 & 3260 & 1314 & 2569 & 0.2761 & 1.7556 & 0.0482 \\
\hline 16 & 10599 & 3162 & 943 & 2925 & 0.3143 & 2.4629 & 0.0604 \\
\hline
\end{tabular}

Table 1: Evaluation of the regularized segments in terms of size of area, perimeter and compactness. The segmentation error with respect to the initial contour is given by the absolute number of wrongly classified pixels, $E_{a b s}$, and the root mean squared error, $E_{\text {mean }}$. The Chamfer distance error evaluation is given by the absolute error $L_{1}$ and the root mean squared error $L_{2}$. The area of the initial segment is 9306 pixels, with perimeter 5458 pixels and compactness 3201 .

Figure 3 shows the original contour as well as the optimized contours for three different levels of scale. The quantitative evaluation of the regularized optimization results are presented in table 1 and show a continuous decrease in segmentation quality, perimeter and compactness. The area is at first slightly decreasing due to loss of detail for increasing $\sigma$, but for very low resolutions (high scales), it is drastically increasing because the contour is blown up in scale. The active contour obviously adjusts better to the object for finer scales, as for coarser scales smaller features are disappearing, thus the snake is attracted by stronger features from far away or even performs short cuts in order to remain elastic. However, points of high curvature tend to be preserved even for large scales due to the enforced adjustment of the snake bending to the underlying isophote image curvature.

\section{Discussion}

We have presented a new and effective method applying an active contour model for shape description, the novelty lying in its integration of multiscale differential 
invariants in terms of minimizing the deviation of the contour curvature from the underlying isophote image intensity curvature and optimizing the model's attraction to the smoothed gradient and the zero-crossings of the Laplacian-of-Gaussian. The resulting hierarchy of shapes for various scales allows to describe an object at multiple resolutions. We evaluated the shape optimization results with simple shape descriptors like the region size, perimeter and compactness, and were able to evaluate the segmentation quality by computing the segmentation error when comparing the optimized shape with the initial object. A special emphasis was put on the rate of change of the shape under change of scale and on the adjustment of the contour curvature to the isophote image curvature. The results were very promising, as most of the chosen shape descriptors showed linear behavior. This novel approach for shape analysis can now be applied not only to perform quantifying measurements on a single shape, but for shape comparisons between different shapes as well.

The presented active contour model for shape description using differential invariants in scale is currently investigated for its clinical relevance in future work, as one major medical task in image processing is the quantification of deformations of the brain in certain diseases such as epilepsy. This ability to produce quantitative shape description metrics for distinguishing between normals and abnormals, as well as matching to atlases, appears to be of crucial importance for clinical applications.

\section{References}

[1] A.A. Amini, Terry E. Weymouth, and Ramesh C. Jain. Using dynamic programming for solving variational problems in vision. IEEE Transaction on Pattern Analysis and Machine Intelligence, 12(9):855-867, September 1990.

[2] R. Bartels, J. Beatty, and B. Barsky. An Introduction to Splines for Use in Computer Graphics and Geometric Modelling. Morgan Kaufmann, Los Altos, CA, 1987.

[3] G. Borgefors. Distance transformations in digital images. Computer Vision, Graphics, and Image Processing, 34:344-371, 1986.

[4] F.S. Cohen and J.-Y. Wang. Modeling image curves using invariant 3-d object curve models - a path to 3-d recognition and shape estimation from image contours. IEEE Transactions on Pattern Analysis and Machine Intelligence, 16(1):1-23, January 1994.

[5] L.D. Cohen and I. Cohen. Finite-element methods for active contour models and balloons for 2-d and 3-d images. IEEE Transactions on Pattern Analysis and Machine Intelligence, 15(11):1131-1147, 1993.

[6] T.F. Cootes, A. Hill, C.J. Taylor, and J. Haslam. The use of active shape models for locating structures in medical imaging. In H.H. Barret and A.F. Gmitro, editors, Information Processing in Medical Imaging, Lecture Notes in Computer Science, pages 33-47. Springer-Verlag, June 1993. 
[7] M. Kass, A. Witkin, and D. Terzopoulos. Snakes: Active contour models. In Proceedings of the 1st International Conference of Computer Vision, London, England, pages 259-268. IEEE, June 1987.

[8] J.J. Koenderink. The structure of images. Biological Cybernetics, 50:363-370, 1984.

[9] T. Lindeberg. On scale selection for differential operators. In Proc. 8th Scandinavian Conference on Image Analysis, pages 857-866, Troms $\varnothing$, Norway, May 1993.

[10] F. Mokhtarian and A.K. Mackworth. Scale-based description and recognition of planar curves and two-dimensional shapes. IEEE Transactions on Pattern Analysis and Machine Intelligence, 8(1):34-43, January 1986.

[11] F. Mokhtarian and A.K. Mackworth. A theory of multiscale, curvaturebased shape representation for planar curves. IEEE Transactions on Pattern Analysis and Machine Intelligence, 14(8):789-805, August 1992.

[12] N. Rougon and F. Prêteux. Directional adaptive deformable models for segmentation with application to $2 \mathrm{~d}$ and $3 \mathrm{~d}$ medical images. Technical report, Télécom Paris, Département Images, 46, Rue Barrault, 75013 Paris, France, 1993.

[13] D. Rueckert and P. Burger. Contour fitting using an adaptive spline model. In Proceedings of the BMVC, 1995.

[14] B.M. ter Haar Romeny, L.M.J. Florack, J.J. Koenderink, and M.A. Viergever. Scale-space: Its natural operators and differential invariants. In A.C.F. Colchester and D.J. Hawkes, editors, Information Processing in Medical Imaging, volume 511 of Lecture Notes in Computer Science, pages 239-255. SpringerVerlag, 1991.

[15] B.M. ter Haar Romeny, L.M.J. Florack, A.H. Salen, and M.A. Viergever. Higher order differential structure of images. In H.H. Barret and A.F. Gmitro, editors, Information Processing in Medical Imaging, Lecture Notes in Computer Science, pages 77-93. Springer-Verlag, June 1993.

[16] D. Terzopoulos, A. Witkin, and M. Kass. Symmetry-seeking models for 3D object reconstruction. In Proceedings of the 1st International Conference on Computer Vision, London, England, pages 269-276, Piscataway, NJ, 1987. IEEE.

[17] D.J. Williams and M. Shah. A fast algorithm for active contours and curvature estimation. CVGIP:Image Understanding, 55(1):14-26, January 1992.

[18] A. Witkin. Scale-space filtering. In Int. Joint Conference on Artificial Intelligence (Karlsruhe, Germany), pages 1019-1022, 1983. 